\title{
Chirurgie de l'arthrose des membres inférieurs chez le sujet âgé
}

Surgery of lower limb for osteoarthritis in the elderly

Charles-Henri Flouzat-Lachaniette ${ }^{1 *}$, David Giber ${ }^{1}$, Claire Bastard $^{1}$, Arnaud Dubory ${ }^{1}$, Florent Eymard $^{2}$, Victor Housset ${ }^{1}$

1. Service de Chirurgie Orthopédique et Traumatologique, Hôpitaux Universitaires Henri Mondor, AP-HP - UPEC, 51 avenue du Maréchal de Lattre de Tassigny - 94010 Créteil Cedex, France

2. Service de Rhumatologie, Hôpitaux Universitaires Henri Mondor, APHP - UPEC, 51 avenue du Maréchal de Lattre de Tassigny - 94010 Créteil Cedex, France

\section{* Auteur correspondant}

Auteur correspondant : Charles-Henri Flouzat-Lachaniette,

Téléphone : +33149812603

Fax : +33149812608

charles-henri.flouzat-lachaniette@aphp.fr 


\title{
Résumé
}

L'arthrose des membres inférieurs s'accompagne de douleurs et d'une gêne fonctionnelle, invalidantes au quotidien. Lorsque le traitement médical est dépassé, la mise en place de prothèses est le traitement de choix chez le sujet âgé. L’objectif de ce travail est de rapporter les spécificités liées au patient, aux techniques opératoires et aux implants dans le traitement chirurgical de l'arthrose des membres chez le sujet âgé.

Les patients âgés ayant bénéficié d'une pose de prothèse totale de hanche ou de genou pour arthose nécessitent souvent plusieurs semaines de récupération. Ils obtiennent d'excellents résultats à long terme au prix d'une hospitalisation plus longue et de taux de complications postopératoires et de mortalité plus élevés. L'adaptation de la stratégie chirurgicale et le type d'implants utilisés doivent être adaptés aux risques spécifiques des sujets âgés. La prise en charge doit intégrer une cogestion gériatrique avant et après la chirurgie afin de réduire le taux de complications mineures.

- Mots-clés : arthrose ; hanche ; genou ; chirurgie ; complications ; sujets âgés ; RAAC ; implants

\begin{abstract}
Osteoarthritis of the lower limbs causes pain and discomfort in daily activities. After medical treatment, knee or hip replacements are the treatment of choice in the elderly. The aim of this work is to report the specificities related to the patient, the surgical techniques and the implants in the surgical treatment of osteoarthritis of the lower limbs in the elderly population.

Elderly patients who had hip or knee replacement for osteoarthritis often require several weeks of recovery. They achieve excellent long-term results at the cost of longer hospitalization and higher rates of postoperative complications and mortality. The surgical strategy and the type of implants used must be adapted to the specific risks of the elderly. Management should include geriatric co-management before and after surgery to reduce the rate of minor complications.
\end{abstract}

- $\quad$ Keywords: osteoarthritis; knee; hip; surgery; complications; elderly population; fast-track; implants 


\section{Introduction}

L'arthrose est une pathologie fréquente qui touche $13 \%$ des femmes et $10 \%$ des hommes audelà de 60 ans [1]. Dans les pays développés, entre le vieillissement de la population et le développement quasi épidémique du nombre de personnes en surpoids, voire obèses, il est probable que ces taux augmentent sur la prochaine décennie. L'arthrose des membres inférieurs s'accompagne de douleurs et d'une gêne fonctionnelle, invalidantes au quotidien. Lorsque le traitement médical est dépassé, la mise en place de prothèses est le traitement de choix chez le sujet âgé. Les arthroplasties des membres inférieurs permettent au patient de récupérer rapidement sa fonction et son autonomie. Il n'y a quasiment pas de place pour les chirurgies conservatrices dans le traitement de l'arthrose du sujet âgé.

Afin de simplifier cette présentation, nous nous limiterons au traitement de la coxarthrose et la gonarthrose qui sont les formes d'arthrose les plus invalidantes au quotidien. Nous avons exclu de notre analyse les sujets âgés opérés d'atteintes rhumatismales, d'ostéonécroses ou de causes traumatiques.

Il a été évalué que la probabilité de bénéficier d'une prothèse totale de hanche (PTH) au cours de la vie d'un individu est de $6-7 \%$ et celle de bénéficier d'une prothèse totale de genou (PTG) est de $10-11 \%$ [2]. Kurtz et al. [3] ont prédit une augmentation de $174 \%$ du nombre de PTH et de $673 \%$ du nombre de PTG implantées d'ici à 2030 aux États-Unis. Logiquement, ces prothèses seront implantées chez des sujets de plus en plus âgés. Le traitement de l'arthrose du sujet âgé est donc un problème majeur de santé publique. L’objectif de ce travail est de rapporter les spécificités liées au patient, aux techniques opératoires et aux implants dans le traitement chirurgical de l'arthrose des membres chez le sujet âgé.

\section{Qu'est-ce qu'un sujet âgé pour un chirurgien orthopédiste ?}

Le bureau américain du recensement définit comme «âgées » les personnes de 65 ans et plus. Beaucoup d'études de la littérature se basent sur cette définition. Mais la définition du « sujet âgé » varie beaucoup d'un article à l'autre (de 70 à 90 ans), ce qui complique la comparaison des résultats. Cependant, il est plus important de parler d'âge physiologique que d'âge chronologique, notamment dans le cadre de la prise en charge chirurgicale de l'arthrose. C'est la fragilité du patient qui définit véritablement son âge. Les patients «âgés » sont ceux présentant des comorbidités nécessitant une prise en charge globale. Outre les tares associées, on retrouve dans cette population une altération des capacités locomotrices, parfois associée à d'autres atteintes sensorielles (visuelle ou auditive), à un risque accru de chute, à de l'ostéoporose ou encore à une diminution de la masse, de la force et de la fonction musculaires, également appelée sarcopénie.

\section{Quand doit-on proposer une PTH ou une PTG à un patient âgé ?}

L'indication opératoire chez le sujet âgé dépend des mêmes paramètres que pour le sujet jeune. Les paramètres les plus importants à prendre en compte sont cliniques : douleur et impotence 
fonctionnelle car ce sont les deux paramètres sur lesquels on peut espérer le gain le plus important dans les suites d'une arthroplastie des membres inférieurs [4-6]. La raideur doit également être évaluée car elle peut rendre techniquement plus complexe l'acte chirurgical ; elle peut notamment entraîner des difficultés pour obtenir une bonne exposition de la hanche ou nécessiter un relèvement de la tubérosité tibiale antérieure en cas de patella basse et fixée, surtout dans les grands valgus. Enfin, il existe une indication particulière d'arthroplastie totale du genou : la grande déformation en valgus, qui se majore au cours du temps, et qui se traduit par une instabilité à la marche, parfois indolore.

Le diagnostic clinique doit bien entendu être étayé par un bilan radiographique confirmant l'atteinte articulaire. Ce bilan permet de confirmer le diagnostic d'arthrose en éliminant les diagnostics différentiels (traumatisme passé inaperçu, rhumatologique inflammatoire, infection, tumeur, etc.) ; il permet également la planification chirurgicale. Il faut cependant garder en tête que les sujets âgés présentent souvent plusieurs pathologies intriquées de l'appareil locomoteur (scoliose lombaire dégénérative et coxarthrose, par exemple) et que l'examen clinique peut être difficile chez eux. En cas de doute, il ne faut pas hésiter à réaliser un test anesthésique pour confirmer quelle articulation est responsable de la majeure partie des symptômes du patient.

\section{Chirurgie conservatrice ou non conservatrice ?}

Il existe deux stratégies pour traiter chirurgicalement l'arthrose des membres inférieurs : les chirurgies conservatrices (représentées essentiellement par les ostéotomies) et les chirurgies non conservatrices (les arthroplasties). Le choix du type de chirurgie dépend de l'âge du patient et de son terrain. Les sujets jeunes ont une demande fonctionnelle importante (activité professionnelle, voire sportive). Les sujets âgés ont une demande différente, souvent inférieure à celle de la population âgée de moins 65 ans ; elle est centrée sur le soulagement de la douleur, l'amélioration de la fonction de l'articulation et le maintien d'un niveau d'autonomie suffisant pour pouvoir effectuer les tâches de la vie courante.

Les ostéotomies visent à modifier l'axe d'un membre afin de modifier la répartition des contraintes au niveau de l'articulation et de ralentir ainsi l'évolution du processus arthrosique. Bien qu'elles permettent de conserver l'articulation native du patient, il s'agit de chirurgies dont les suites opératoires sont plus lourdes, avec parfois un appui différé le temps de la consolidation osseuse. La restauration de la fonction est donc plus longue et ce type de chirurgie est réservé aux sujets jeunes. Les arthroplasties sont préférées chez le sujet âgé car elles permettent un appui immédiat et une récupération fonctionnelle plus simple et plus rapide. On peut alors séparer les arthroplasties totales des arthroplasties partielles, comme les prothèses unicompartimentales (PUC) du genou remplaçant un seul des trois compartiments du genou (fémoro-tibial médial, latéral ou fémoro-patellaire). Les données à prendre en compte sont : la balance bénéfice/risque, la durée de vie limitée des implants et éventuellement l'extension du processus arthrosique, pour les PUC.

\section{Jusqu’à quel âge peut-on proposer une PTH ou une PTG ?}

C'est une question qui se pose régulièrement en consultation. Le patient âgé est parfois considéré comme « trop âgé » pour une chirurgie « trop risquée ». Bref, il est « trop tard pour opérer ». 
En pratique, $45 \%$ des patients non opérés déclarent que la chirurgie ne leur a jamais été proposée comme un traitement potentiel [7]. Il est vrai que le risque de complication est beaucoup plus élevé chez les sujets de plus de 80 ans que chez les patients plus jeunes [7, 8]. Cela doit être mis dans la balance risque/bénéfice. Cependant, même s’ils mettent plusieurs semaines à récupérer, les patients âgés ayant eu une arthroplastie de la hanche ou du genou obtiennent d'excellents résultats à long terme $[7,8]$. Il est donc judicieux de discuter de la chirurgie tout en avertissant les patients âgés et leurs proches des risques accrus dans ce groupe d'âge [8]. Certains préféreront prendre le risque de la chirurgie plutôt que de devoir continuer à vivre avec un handicap fonctionnel qu'ils considèrent intolérable.

Il faut noter le cas particulier des patients ayant « loupé le coche » de la chirurgie à un âge raisonnable, du fait de leurs propres réticences, de celles de leur médecin traitant ou des chirurgiens qu'ils ont pu consulter. Leur arthrose s'est majorée à un point tel qu'ils circulent en fauteuil roulant (hanches) ou que la déformation de leur membre inférieur est devenue majeure (genoux). Ils envisagent désormais une chirurgie à un âge beaucoup plus tardif, ce qui entraîne une majoration du risque opératoire [8]. La chirurgie du genou est également techniquement plus difficile sur les grandes désaxation et peut nécessiter le recours à une prothèse à charnière. Cependant, s'il a pu être démontré que ces patients âgés présentant de grandes déformations pouvaient plus souvent recourir à une aide technique à la marche en postopératoire, à deux ans de recul, il n'y avait pas de différence significative en termes de fonction, de douleur ou de force [9].

\section{Quel est le taux de mortalité après une arthroplastie du membre inférieur ?}

En utilisant les données nationales sur les patients hospitalisés entre 1990 et 2004, Memtsoudis et al. [10] ont retrouvé un taux cumulatif de mortalité hospitalière de $0,35 \%$ sur environ 6 901324 procédures (PTH et PTG). Les facteurs de risque indépendants les plus importants étaient l'embolie pulmonaire et les complications neuro-vasculaires, qui multipliaient par 40 le risque de décès. Les facteurs de risque préopératoires de mortalité à l'hôpital étaient la reprise d'arthroplastie totale de la hanche, l'âge avancé et la présence de comorbidités comme la démence, les maladies rénales et neurovasculaires.

Pour ce qui est de la mortalité à court terme plus spécifiquement, une étude sur les prothèses totales de hanche et de genou chez les nonagénaires a montré un taux de mortalité de $0,9 \%$ dans les trente premiers jours postopératoires, ce qui était significativement plus important que chez la population de sujet plus jeune $(0,2 \%)$ [11]. Des résultats similaires ont été retrouvés notamment par Maempel et al. [12] chez des patients de plus de 80 ans opérés pour une prothèse totale de genou avec un taux de décès de $0,86 \%$ à un mois et de $3,2 \%$ à un an contre respectivement $0,15 \%$ et $1,5 \%$ chez les moins de 75 ans.

Quand on se pose la question de la mortalité postopératoire des sujets âgés, il est nécessaire de prendre en compte les paramètres de mortalité globale de cette population. Une étude portant sur les nonagénaires ayant été opérés d'une PTH a montré que, malgré un taux de mortalité supérieur chez ces patients par rapport à des patients plus jeunes, celui-ci reste en accord avec le taux de mortalité global attendu de cette population à risque [13]. Ce résultat est confirmé par plusieurs études portant sur la 
mortalité postopératoire des sujets âgés [14, 15]. Le principal soucis dans l'analyse de ce paramètre est que la plupart des études rapportant les taux de mortalités postopératoires ont un faible recul. En effet, du fait du terrain et des comorbidités associées, il est parfois difficile de pouvoir attribuer le décès du patient uniquement à l'acte chirurgical [16].

L'ensemble de ces résultats reste cependant à pondérer. En effet, il a été possible de démontrer qu'en comparant les patients de plus de 80 ans hospitalisés, toutes causes confondues, ceux qui étaient hospitalisés pour une arthroplastie totale de hanche avaient un taux de mortalité inférieur du fait du processus de sélection et d'évaluation préopératoires [17, 18]. Le taux de mortalité passait de $12 \%$ à 5,5\% à 1 an, soulignant l'importance de la sélection préopératoire des patients avant ce type de chirurgie.

\section{Quels sont les risques et les taux de complications à court terme ?}

D'Apuzzo et al. [19], sur un recueil national portant sur plus de huit millions d'arthroplasties de hanche et de genou a pu montrer chez les patients nonagénaires, une multiplication par 2 en postopératoire du nombre de complications cardiaques (Odds Ratio $[\mathrm{OR}]=2,52$ ), du système nerveux central ( $\mathrm{OR}=2,1)$, ou encore respiratoire $(\mathrm{OR}=1,91)$. Ils n'ont cependant pas retrouvé de différence dans les complications chirurgicales (comme les problèmes cicatriciels). Certaines complications liées à l'arthroplastie, comme les luxations, peuvent cependant être plus fréquentes chez les personnes âgées à cause de la sarcopénie et de la présence d'éventuelles comorbidités neurologiques [20]. Le risque d'ischémie aiguë après une arthroplastie totale du genou est faible (0,17 \% dans une série de 4097 patients) [21]. Il s'agit principalement d'une thrombose de l'artère poplitée. Ce risque est majoré chez les personnes plus âgées souffrant d'artériosclérose et peut conduire à une amputation si la complication est diagnostiquée tardivement.

Le taux de mortalité durant l'hospitalisation était de $2,9 \%$, ce qui était onze fois plus important par rapport à la population plus jeune. Une autre étude portant sur plus de 25000 patients octogénaires a retrouvé, par rapport à une population plus jeune, que ces patients avaient une majoration de leur risque d'infarctus du myocarde $(\mathrm{OR}=2,7)$ et de pneumonie $(\mathrm{OR}=3,5)$ [22]. Les risques de confusion et de problèmes urinaires étaient également majorés.

D'autres travaux présentent cependant des résultats moins péjoratifs sur le risque de complications à court terme et ne retrouvent pas de différence entre les populations « jeunes » et « âgées » après arthroplastie du genou [23, 24].

\section{Quels sont les bénéfices et les résultats fonctionnels des arthroplasties du membre inférieur?}

Nous pouvons regretter dans la littérature une grande disparité dans la sélection des patients, ainsi que dans l'analyse des résultats des PTH et des PTG chez les sujets âgés.

Les arthroplasties de hanche sont bien connues désormais pour être celles qui ont les résultats les plus fiables et reproductibles à court terme [6]. Leur objectif biomécanique est de permettre de restaurer un axe de rotation le plus proche de celui de l'anatomie native du patient afin de reproduire au mieux la cinématique de la hanche. Dans une étude prospective, Clément et al. [4] ont montré qu'il n’y 
avait pas de différence significative dans l'amélioration moyenne des scores de hanche en fonction de l'âge à 12 mois (Score de Oxford autour de 20/48 dans les 2 groupes). Les sujets jeunes avaient une amélioration significativement plus importante de la composante bien-être physique du score SF-12, mais le groupe le plus âgé était plus satisfait de sa prothèse. Le groupe le plus âgé avait une durée d'hospitalisation plus longue (5,9 jours contre 9,0 jours) et des taux de complications postopératoires et de mortalité plus élevés [4].

Les arthroplasties du genou ont pour réputation d'être des chirurgies plus lourdes que les arthroplasties de hanche, aussi bien pour le risque de saignement que pour la longueur des suites opératoires. Elles ont pour objectif de reproduire l'anatomie du genou natif dans toute sa complexité. La reproduction de la cinématique naturelle du genou est beaucoup plus difficile car elle doit reproduire les mouvements de flexion/extension, de rotation et de roulement/glissement. D'ailleurs, les résultats fonctionnels sont souvent moins bons pour les PTG que pour les PTH [25]. Elles restent néanmoins des interventions chirurgicales sûres chez les sujets âgés, même chez les plus de 90 ans [26]. Des résultats similaires sur la douleur et sur la fonction ont pu être rapportés chez les nonagénaires après PTH et PTG, mais la diminution de la douleur était trois fois plus marquée après les PTG [5]. Aucune différence significative n'a été observée dans l'amélioration moyenne des scores d'Oxford genou selon l'âge à 12 mois [4]. Le score de satisfaction moyen était de 84,9\% dans tous les groupes d'âge sauf chez les moins de 55 ans (78,3\%) [27]. Le groupe le plus âgé avait une durée d'hospitalisation plus longue (6,2 jours contre 8,3 jours) et des taux de complications postopératoires et de mortalité plus élevés [4].

Plus récemment, Murphy et al. [28], dans une revue de la littérature portant sur 32 articles, ont également conclu que ces chirurgies avaient des résultats satisfaisants chez les patients âgés, malgré la nécessité pour le chirurgien de prendre en compte un risque majoré de complications, une durée de séjour prolongée et un taux de mortalité plus important. En analysant les résultats fonctionnels et la qualité de vie liée à la santé sur une grande série de patients ayant été opérés de PTH ou de PTG, Jones et al. [29, 30] ont conclu que la douleur, la fonction, la vitalité et la santé en général étaient significativement améliorées 6 mois après le remplacement de l'articulation et que l'âge n'était pas un déterminant significatif de la douleur ou de la fonction. En outre, Chang et al. [31] ont conclu que la PTH permettait de réduire les coûts ou, dans le pire des cas, d'améliorer de manière rentable l'espérance de vie ajustée en fonction de la qualité de vie, à court et à long terme. La PTG apporte un soulagement de la douleur, une satisfaction et une amélioration de la qualité de vie pour les patients octogénaires au même niveau que des patients septuagénaires [32].

\section{Faut-il une technique chirurgicale et des implants spécifiques pour les sujets}

\section{âgés ?}

Le choix de la technique et des implants vise à réduire le risque de complications périopératoires tout en permettant un bon résultat fonctionnel. Chaque technique et chaque implant possèdent des avantages et des inconvénients qu'il convient de mettre dans la balance bénéfice/risque avec le terrain du patient. D’une façon générale, pour optimiser la prise en charge des patients âgés, il 
faut privilégier les voies d'abord les moins invasives, en étant plus anatomique et en cherchant à préserver le plus possible les structures musculo-tendineuses périarticulaires.

PTH, risque de luxation et ostéoporose. Le choix des implants et la technique opératoire sont dictés par les habitudes du chirurgien, mais également par les spécificités de chaque patient. Par exemple, l'ostéoporose augmente le risque fracturaire périopératoire et la sarcopénie contribue à augmenter le risque de luxation. Il est donc nécessaire au moment de la planification préopératoire de recourir à une stratégie chirurgicale adaptée lors de l'implantation d'une PTH. Le sujet âgé est, de facto, plus à risque de présenter des accidents d'instabilité par rapport aux sujets plus jeunes à cause de la sarcopénie et de la présence d'éventuelles comorbidités neurologiques [20]. Les facteurs permettant de limiter le risque de luxation sont les suivants : l'abord chirurgical, la restauration de l'offset et de la longueur du membre inférieur opéré et le choix de l'implant.

La voie d'abord postérieure est la voie d'abord la plus communément utilisée par les chirurgiens pour implanter une PTH mais on lui reproche un taux plus élevé de luxation car elle nécessite la section des muscles pelvitrochantériens, stabilisateurs de hanche. La voie d'abord antérieure, quant à elle, est utilisée de manière moins fréquente (20\% des cas, mais ce taux est en augmentation sur la dernière décennie). C'est une voie dite « anatomique » qui permet d'accéder à la hanche en passant en intermusculaire et donc en épargnant les structures musculo-tendineuses périarticulaires. C'est l'une des raisons expliquant l'engouement récent des chirurgiens et des patients pour cette voie d'abord. En pratique, peu d'études ont comparé les différentes voies d'abord ; le taux de luxation en fonction de la voie d'abord utilisée semble globalement similaire entre les différentes techniques [33]. Il faut noter que l'exposition du fémur est plus difficile par voie antérieure. Ceci expose à un risque plus élevé de fracture fémorale, particulièrement chez les femmes et les patients obèses [34].

Outre l'abord chirurgical, afin de minimiser le risque de luxation, le chirurgien tente de restaurer au mieux la longueur du membre inférieur opéré et l'offset fémoral, c'est-à-dire le bras de levier entre le centre de la hanche et le grand trochanter, en garantissant ainsi une tension musculaire abarticulaire optimale autour de l'implant. Cela passe par une planification préopératoire rigoureuse.

Le choix de l'implant est également important pour réduire le risque de luxation. L'utilisation de têtes fémorales de grand diamètre permettant d'augmenter le diamètre de débattement de la tête avant que celle-ci ne puisse se luxer par conflit du col fémoral et de la cupule acétabulaire lors des mouvements de grande amplitude de la hanche [35]. Le cotyle à double mobilité, composé de deux cupules se mobilisant l'une dans l'autre, a largement permis de diminuer le risque de luxation tout en permettant de maintenir, voire d'augmenter les amplitudes articulaires de la hanche [20]. Le cotyle rétentif est équipé d'une bague mobile intérieure armée venant retenir la tête fémorale prothétique fixée au niveau de la tige fémorale afin de prévenir des phénomènes de luxation [20] (Figure 1).

Implants cimentés ou sans ciment. Le recours aux prothèses non cimentées est de plus en plus privilégié chez les sujets jeunes lors de l'implantation des PTH mais également des PTG. Cette technique consiste à impacter en force des implants de taille suffisamment importante pour obtenir une 
stabilité primaire ; la stabilité secondaire est obtenue via le processus biologique d'ostéointégration qui nécessite une repousse osseuse sur le revêtement de surface de l'implant. Cette technique offre un gain de temps puisqu'elle permet de s'affranchir du temps de polymérisation du ciment acrylique ; elle limite également le risque de descellement. Cependant, cette stratégie est souvent évitée chez les patients plus âgés, souvent ostéoporotiques, du fait du risque majoré de fracture peropératoire passant de $1 \%$ (prothèses cimentées) à près de $5 \%$ (prothèses impactées) [36].

Les prothèses cimentées permettent de nous affranchir de ce risque. Le ciment (polyméthacrylate de méthyl) va polymériser à l'interface entre l'os et la prothèse afin de servir de joint, permettant une stabilité immédiate de l'implant. Le risque principal de ce type de technique est le « choc au ciment », caractérisé par une hypoxie et/ou une hypotension durant les périodes avoisinant le temps du cimentage et l'insertion de la prothèse [37]. Peu d'études se sont concentrées sur cette question dans le traitement de la coxarthrose, mais le cimentage des implants est recommandée par une étude ayant évalué les résultats des prothèses dans le traitement des fractures du col du fémur chez des patients par définition ostéoporotiques [38]. Cette étude a retrouvé un taux de complications très significativement supérieur dans le groupe des prothèses non cimentées par rapport aux prothèses cimentées.

Au total, le choix du type d'ancrage de l'implant nécessite une évaluation préopératoire de la fragilité osseuse et le recours aux prothèses cimentées doit être privilégié chez les patients ostéoporotiques après analyse de la balance bénéfice/risque du cimentage en fonction des antécédents cardiaques.

PUC vs. PTG. Les PUC permettent de réaliser une chirurgie mini-invasive en préservant une partie de l'articulation native. Siman et al. [39] ont d'ailleurs démontré que, chez les sujets de plus de 75 ans, l'utilisation d'une PUC permettait une récupération initiale plus précoce qu'après une PTG avec, au recul de 2 ans, aucune de différence significative en termes de complication. Les indications de PUC chez les sujets âgés sont limitées car, dans cette population, l'atteinte arthrosique touche généralement plus d'un compartiment ou il existe une atteinte du pivot central. Dans les bonnes indications de PUC, il faut prendre en compte deux risques : celui de l'évolution de la maladie arthrosique vers un autre compartiment et celui d'un enfoncement de l'implant en cas d'ostéoporose.

PTG et ostéoporose. Il est recommandé actuellement de recourir à l'utilisation de quilles d'extension au tibia et/ou au fémur, en cas de fragilité osseuse afin de permettre d'augmenter la stabilité de l'implant, son ancrage osseux et de limiter le risque d'enfoncement ou de descellement précoce (Figure 2). Leur utilisation a montré des résultats fonctionnels aussi bons que les implants sans quilles à un recul de 10 ans [40].

\section{Y a-t-il nécessité d'une prise en charge pluridisciplinaire associant un orthogériatre au chirurgien orthopédiste ? Quelle préparation préopératoire peut-on envisager ?}


La chirurgie du sujet âgé présentant plus de comorbidités entraîne une surmortalité et une augmentation du risque de complications par rapport aux sujets jeunes. La durée d'hospitalisation est également augmentée dans ce groupe de patient [4]. En postopératoire immédiat, 3 risques sont à surveiller particulièrement : le saignement, les chutes et les décompensations de tares.

Sehat et al. [41] ont montré que la perte totale sanguine moyenne rapportée au cours de la réalisation d'une PTH ou d'une PTG est de 1,5 L. Une grande partie de celle-ci est constituée d'une perte cachée (donc non recueillie en peropératoire ou dans les drainages postopératoires). Celle-ci est significativement plus importante pour les PTG que pour les PTH (respectivement $49 \%$ du total contre $26 \%$ ). Il est donc important de rester vigilant dans les suites d'une chirurgie prothétique, notamment après une PTG où le saignement est sous-estimé. De plus, beaucoup de sujets âgés prennent en préopératoire des traitements antiagrégants plaquettaires ou anticoagulants pour des pathologies cardiovasculaires associées, ce qui fait craindre au chirurgien une majoration du saignement. Dans les faits, les études sur le sujet ne semblent pas en faveur d'un risque majoré de saignement chez les sujets les plus âgés par rapport à des cohortes de sujets plus jeunes [42]. Le risque d'hématome n'est pas majoré par l'âge avancé du patient [43]. Cependant, il semble essentiel chez ces sujets fragiles d'anticiper au maximum le risque hémorragique, que cela soit sur le contrôle du taux d'hémoglobine préopératoire, en anticipant le recours à une transfusion sanguine ou encore en utilisant en peropératoire des agents antifibrinolytiques.

Il existe un taux plus important de fractures périprothétiques chez les sujets de plus de 80 ans, à la fois pour les PTH et les PTG [44]. Jørgensen et al. [45] ont montré que 1,6\% des patients chutant après la mise en place d'une PTH ou d'une PTG nécessitaient une hospitalisation, 54,8 \% de ces chutes survenaient au cours des premiers mois postopératoires. Il est donc nécessaire de mettre en place des mesures précoces et spécifiques visant à la prévention des chutes chez les patients âgés bénéficiant d'une arthroplastie de hanche ou de genou. Cette prévention se doit d'être renforcée chez patients présentant un antécédent de chute.

Bien qu'il n'y ait pas de consensus quant à la réalisation d'une prise en charge pluridisciplinaire en amont de la réalisation d'une arthroplastie des membres inférieurs, de nombreux travaux soulignent l'amélioration des résultats suite à une telle prise en charge ; c'est de là qu'est né le concept d'orthogériatrie qui a montré son impact sur le pronostic des patients dans le cadre des fractures de l'extrémité supérieure du fémur, en diminuant les taux de mortalité et de complications périopératoires [46]. En effet, la gestion des patients âgés et de leurs comorbidités bénéficie d'une prise en charge spécifique gériatrique permettant de diminuer le taux de complications, la durée de séjour et le taux de réhospitalisation (de 4,4\% contre 1,8\%) [47]. Une prise en charge orthogériatrique dès le préopératoire semble plus susceptible de permettre un retour plus précoce à la maison de ces patients en postopératoire par rapport à ceux vus pour la première fois en postopératoire [47, 48]. Ces consultations gériatriques préopératoires, souvent couplées avec les consultations d'anesthésie, permettent d'anticiper les problèmes médicaux et sociaux postopératoires. Ces consultations permettent, par exemple, de prévenir le risque d'anémie postopératoire. Il n'y a cependant pas de consensus clair sur la meilleure façon de prévenir cette complication. Il semble cependant que le 
recours à l'érythropoïétine semble préférable à la prescription de fer par voie orale ou intraveineuse sur la restauration du stock en fer [49].

En dehors de la prise en charge rhumatologique, chirurgicale, anesthésique ou encore gériatrique de ces patients âgés, il est indispensable d'évoquer le rôle du kinésithérapeute.

La question de la réalisation d'une kinésithérapie préopératoire est toujours débattue. Des travaux ont montré un intérêt de celle-ci sur les résultats fonctionnels postopératoires, que ce soit par la réalisation d'un protocole de rééducation standard pendant les six semaines précédent l'acte chirurgical [50] ou par la réalisation d'un programme de rééducation préopératoire intensive [51]. Mais ces programmes sont souvent mal supportés chez les patients âgés et douloureux. Leur réalisation est donc discutable, d'autant qu'une méta-analyse de 22 études, parue en 2016, concluait que malgré une légère amélioration en termes de douleur et de fonction postopératoire à court terme, les effets de la rééducation étaient trop faibles pour être considérés comme cliniquement efficaces. Ils étaient sans conséquences sur les coûts, la qualité de vie ou encore la durée d'hospitalisation [52]. En postopératoire, en, revanche, la réalisation d'une rééducation précoce a montré, dans une autre métaanalyse récente, une diminution de la durée totale d'hospitalisation, ainsi que du coût dans les suites d'une prothèse totale de hanche ou de genou, sans pour autant augmenter le risque de complications [53].

\section{Les patients âgés sont-ils éligibles aux programmes de Récupération Accélérée Après Chirurgie (RAAC) ou à la chirurgie en ambulatoire ?}

Les programmes de RAAC, inscrits dans les projets d'établissement, ont été initialement développés afin d'optimiser la récupération fonctionnelle des patients après la chirurgie, via une approche globale du patient. Ils tendent à devenir actuellement la prise en charge de référence. Les sujets âgés constituent probablement la catégorie de patients ayant le plus de bénéfice à tirer de cette prise en charge pluridisciplinaire (médecins, kinésithérapeutes, infirmiers, aides soignants et diététiciens) ayant comme objectif commun de permettre au patient de bénéficier d'une hospitalisation plus courte et plus confortable [54]. Les protocoles mettent en place une analgésie multimodale, privilégiant l'association anesthésiques locaux à l'anesthésie générale, des procédures se devant d'être les moins agressives possibles et une réévaluation permanente de chaque composante du programme de réhabilitation.

En postopératoire, après 1 à 3 jours, les patients peuvent retourner à leur domicile, en fonction de leur entourage familial. En effet, il a été démontré que les patients bénéficiant d'une kinésithérapie postopératoire à domicile présentaient des résultats fonctionnels similaires à ceux ayant effectué celleci au sein d'un centre dé rééducation [55]. Il faut cependant prendre en compte le risque d'isolement social du patient. Une étude récente a montré, sur une cohorte de 393 patients ayant bénéficié d'une PTH ou d'une PTG pour arthrose, une augmentation significative du taux d'isolement des patients lors de la phase de rééducation postopératoire en comparaison au préopératoire [56]. Il faut donc intégrer cette donnée dans la prise en charge des patients. 
Sur le principe, l'âge chronologique n'est pas une contre-indication à la chirurgie ambulatoire. Ce sont généralement les comorbidités des patients âgés ou leur isolement qui contre-indiquent ce type de prise en charge. La quête de la chirurgie ambulatoire ne doit pas selon nous être un objectif absolu de la prise en charge postopératoire des sujets âgés après une chirurgie prothétique des membres inférieurs. Une prise en charge raisonnée de ces patients reste indispensable (surveillance des comorbidités) sans pour autant empêcher les programmes de RAAC chez eux.

\section{Quel bilan pré-anesthésique doit-on envisager?}

Les examens complémentaires nécessaires avant chirurgie prothétique des membres inférieurs sont guidés par l'évaluation clinique préopératoire. L'arthroplastie des membres inférieurs est classée en anesthésie comme une chirurgie à risque intermédiaire. Le bilan systématique est rapporté dans le Tableau 1. Un hémogramme est recommandé afin de détecter une éventuelle anémie préopératoire car cette dernière augmente la mortalité postopératoire si elle n'est pas prise en charge préalablement. La réalisation systématique d'un examen cytobactériologique des urines n'est pas justifiée, le dépistage des colonisations urinaires n'ayant aucune incidence sur la prévention des infections du site opératoire. La vérification préopératoire de l'état dentaire doit probablement reposer sur une évaluation clinique préalable. La réalisation d'un tel bilan ne permet pas de réduire le risque de survenue d'infection précoce [57]. Les experts de la Société Française d'Anesthésie et de Réanimation sur l'anesthésie du sujet âgé recommandent en 2017 de calculer le score de Lee [58] afin de déterminer si un électrocardiogramme est suffisant ou si des investigations supplémentaires sont nécessaires (dosage de biomarqueurs et/ou test cardiopulmonaire si score de Lee > 1). Intuitivement, il paraît raisonnable d'effectuer une consultation cardiologique en préopératoire d'une arthroplastie du membre inférieur chez le sujet âgé. En fonction des données de cette consultation, d'autres examens pourront être prescrits (Tableau 1).

Enfin, il est essentiel de connaître et éventuellement d'adapter le traitement des patients ; en effet, certains médicaments doivent être arrêtés ou substitués en préopératoire (Tableau 1).

\section{Conclusion}

Les questions autour de la chirurgie de l'arthrose du genou et de la hanche chez les sujets âgés sont largement étudiées actuellement du fait de l'évolution démographique [3]. Comme Murphy et al. [28], on peut regretter le fait que les études sur le sujet présentent des méthodologies trop différentes avec notamment des cohortes de patients trop hétérogènes et des critères d'évaluation trop variés, rendant leur analyse et leur comparaison difficiles.

Bien que les taux de morbidité et de mortalité soient plus élevés chez les patients âgés, les résultats fonctionnels, le soulagement de la douleur et la satisfaction des patients sont indépendants de l'âge $[4,7,8,28,47]$. L'âge ne doit pas représenter une contre indication à la réalisation d'une arthroplastie de hanche ou du genou. Cependant, pour améliorer la sécurité périopératoire des patients, les chirurgiens orthopédistes doivent intégrer les risques et connaître les résultats cliniques qu'ils peuvent espérer chez un patient âgé [47]. Les chirurgiens doivent informer les patients âgés et leurs 
familles des risques accrus de morbidité et de mortalité. Ils doivent également planifier la chirurgie en fonction des éléments médicaux et sociaux du patient dont ils disposent. Le choix des implants et de l'abord chirurgical est primordial et se conçoit après avoir anticipé toutes les comorbidités que ces sujets âgés peuvent présenter.

Une prise en charge pluridisciplinaire est indispensable avec au minimum les anesthésistes, les cardiologues, et les kinésithérapeutes, idéalement dans le cadre d'un programme de type « RAAC ». Les programmes de RAAC semblent être une avancée majeure dans la prise en charge chirurgicale de l'arthrose de membres inférieurs chez les sujets âgés, mais ils doivent intégrer une cogestion gériatrique avant et après une arthroplastie totale de la hanche ou du genou afin de réduire le taux de complications mineures et la durée des séjours à l'hôpital chez les patients âgés [47, 48].

\section{Conflit d'intérêt}

Les auteurs déclarent n'avoir aucun conflit d'intérêt concernant ce travail. 


\section{Références}

[1] Zhang Y, Jordan JM. Epidemiology of osteoarthritis. Clin Geriatr Med 2010;26(3):355-69.

[2] Culliford DJ, Maskell J, Kiran A, Judge A, Javaid MK, Cooper C, et al. The lifetime risk of total hip and knee arthroplasty: results from the UK general practice research database. Osteoarthritis Cartilage 2012;20(6):519-24.

[3] Kurtz S, Ong K, Lau E, Mowat F, Halpern M. Projections of primary and revision hip and knee arthroplasty in the United States from 2005 to 2030. J Bone Joint Surg Am 2007;89(4):780-5.

[4] Clement ND, MacDonald D, Howie CR, Biant LC. The outcome of primary total hip and knee arthroplasty in patients aged 80 years or more. J Bone Joint Surg Br 2011;93(9):1265-70.

[5] Berend ME, Thong AE, Faris GW, Newbern G, Pierson JL, Ritter MA. Total joint arthroplasty in the extremely elderly: hip and knee arthroplasty after entering the 89th year of life. J Arthroplasty 2003;18(7):817-21.

[6] Gould VC, Blom AW, Wylde V. Long-term patient-reported outcomes after total hip replacement: comparison to the general population. Hip Int 2012;22(2):160-5.

[7] Hamel MB, Toth M, Legedza A, Rosen MP. Joint replacement surgery in elderly patients with severe osteoarthritis of the hip or knee: decision making, postoperative recovery, and clinical outcomes. Arch Intern Med 2008;168(13):1430-40.

[8] Newington DP, Bannister GC, Fordyce M. Primary total hip replacement in patients over 80 years of age. J Bone Joint Surg Br 1990;72(3):450-2.

[9] Zicat B, Rorabeck CH, Bourne RB, Devane PA, Nott L. Total knee arthroplasty in the octogenarian. J Arthroplasty 1993;8(4):395-400.

[10] Memtsoudis SG, Della Valle AG, Besculides MC, Esposito M, Koulouvaris P, Salvati EA. Risk factors for perioperative mortality after lower extremity arthroplasty: a population-based study of 6,901,324 patient discharges. J Arthroplasty 2010;25(1):19-26.

[11] Jauregui JJ, Boylan MR, Kapadia BH, Naziri Q, Maheshwari AV, Mont MA. Total Joint Arthroplasty in Nonagenarians: What Are the Risks? J Arthroplasty 2015;30(12):2102-5.e1.

[12] Maempel JF, Riddoch F, Calleja N, Brenkel IJ. Longer hospital stay, more complications, and increased mortality but substantially improved function after knee replacement in older patients. Acta Orthop 2015;86(4):451-6.

[13] Miric A, Inacio MC, Kelly MP, Namba RS. Are Nonagenarians Too Old For Total Hip Arthroplasty? An Evaluation of Morbidity and Mortality Within a Total Joint Replacement Registry. J Arthroplasty 2015;30(8):1324-7.

[14] Easterlin MC, Chang DG, Talamini M, Chang DC. Older age increases short-term surgical complications after primary knee arthroplasty. Clin Orthop Relat Res 2013;471(8):2611-20.

[15] Belmont PJ, Goodman GP, Hamilton W, Waterman BR, Bader JO, Schoenfeld AJ. Morbidity and mortality in the thirty-day period following total hip arthroplasty: risk factors and incidence. J Arthroplasty 2014;29(10):2025-30.

[16] Murphy SL, Xu J, Kochanek KD. Deaths: final data for 2010. Natl Vital Stat Rep 2013;61(4):1-117. 
[17] Ferrer A, Formiga F, Ruiz D, Mascaró J, Olmedo C, Pujol R. Predictive items of functional decline and 2-year mortality in nonagenarians--the NonaSantfeliu study. Eur J Public Health 2008;18(4):406-9.

[18] Formiga F, Ferrer A, Mascaró J, Ruiz D, Olmedo C, Pujol R. Predictive items of one-year mortality in nonagenarians. The NonaSantfeliu Study. Aging Clin Exp Res 2007;19(4):265-8.

[19] D'Apuzzo MR, Pao AW, Novicoff WM, Browne JA. Age as an independent risk factor for postoperative morbidity and mortality after total joint arthroplasty in patients 90 years of age or older. $\mathrm{J}$ Arthroplasty 2014;29(3):477-80.

[20] Hernigou P, Trousselier M, Roubineau F, Bouthors C, Flouzat Lachaniette CH. Dual-mobility or Constrained Liners Are More Effective Than Preoperative Bariatric Surgery in Prevention of THA Dislocation. Clin Orthop Relat Res 2016;474(10):2202-10.

[21] Calligaro KD, DeLaurentis DA, Booth RE, Rothman RH, Savarese RP, Dougherty MJ. Acute arterial thrombosis associated with total knee arthroplasty. J Vasc Surg 1994;20(6):927-30; discussion $30-2$.

[22] Kreder HJ, Berry GK, McMurtry IA, Halman SI. Arthroplasty in the octogenarian: quantifying the risks. J Arthroplasty 2005;20(3):289-93.

[23] Biau D, Mullins MM, Judet T, Piriou P. Is anyone too old for a total knee replacement? Clin Orthop Relat Res 2006;448:180-4.

[24] Seo JG, Moon YW, Cho BC, Kim SC, Ko YH, Jang SP, et al. Is Total Knee Arthroplasty a Viable Treatment Option in Octogenarians with Advanced Osteoarthritis? Knee Surg Relat Res 2015;27(4):221-7.

[25] Skinner D, Tadros BJ, Bray E, Elsherbiny M, Stafford G. Clinical outcome following primary total hip or knee replacement in nonagenarians. Ann R Coll Surg Engl 2016;98(4):258-64.

[26] Joshi AB, Gill G. Total knee arthroplasty in nonagenarians. J Arthroplasty 2002;17(6):681-4.

[27] Williams DP, Price AJ, Beard DJ, Hadfield SG, Arden NK, Murray DW, et al. The effects of age on patient-reported outcome measures in total knee replacements. Bone Joint J 2013;95-B(1):3844.

[28] Murphy BPD, Dowsey MM, Choong PFM. The Impact of Advanced Age on the Outcomes of Primary Total Hip and Knee Arthroplasty for Osteoarthritis: A Systematic Review. JBJS Rev 2018;6(2):e6.

[29] Jones CA, Voaklander DC, Johnston DW, Suarez-Almazor ME. Health related quality of life outcomes after total hip and knee arthroplasties in a community based population. J Rheumatol 2000;27(7):1745-52.

[30] Jones CA, Voaklander DC, Johnston DW, Suarez-Almazor ME. The effect of age on pain, function, and quality of life after total hip and knee arthroplasty. Arch Intern Med 2001;161(3):454-60.

[31] Chang RW, Pellisier JM, Hazen GB. A cost-effectiveness analysis of total hip arthroplasty for osteoarthritis of the hip. JAMA 1996;275(11):858-65.

[32] Lizaur-Utrilla A, Martinez-Mendez D, Miralles-Muñoz FA, Marco-Gomez L, Lopez-Prats FA. Risk-Benefit on Quality of Life After Total Knee Arthroplasty in Octogenarians. J Arthroplasty 2017;32(8):2417-20. 
[33] Kwon MS, Kuskowski M, Mulhall KJ, Macaulay W, Brown TE, Saleh KJ. Does surgical approach affect total hip arthroplasty dislocation rates? Clin Orthop Relat Res 2006;447:34-8.

[34] Hartford JM, Knowles SB. Risk Factors for Perioperative Femoral Fractures: Cementless Femoral Implants and the Direct Anterior Approach Using a Fracture Table. J Arthroplasty 2016;31(9):2013-8.

[35] Blakeney WG, Beaulieu Y, Puliero B, Lavigne M, Roy A, Massé V, et al. Excellent results of large-diameter ceramic-on-ceramic bearings in total hip arthroplasty. Bone Joint J 2018;100$\mathrm{B}(11): 1434-41$.

[36] Gruner A, Hockertz T, Reilmann H. [Periprosthetic fractures: classification, management, therapy]. Unfallchirurg 2004;107(1):35-49.

[37] Donaldson AJ, Thomson HE, Harper NJ, Kenny NW. Bone cement implantation syndrome. Br J Anaesth 2009;102(1):12-22.

[38] Chammout G, Muren O, Laurencikas E, Bodén H, Kelly-Pettersson P, Sjöö H, et al. More complications with uncemented than cemented femoral stems in total hip replacement for displaced femoral neck fractures in the elderly. Acta Orthop 2017;88(2):145-51.

[39] Siman H, Kamath AF, Carrillo N, Harmsen WS, Pagnano MW, Sierra RJ. Unicompartmental Knee Arthroplasty vs Total Knee Arthroplasty for Medial Compartment Arthritis in Patients Older Than 75 Years: Comparable Reoperation, Revision, and Complication Rates. J Arthroplasty 2017;32(6):1792-7.

[40] Kolisek FR, Mont MA, Seyler TM, Marker DR, Jessup NM, Siddiqui JA, et al. Total knee arthroplasty using cementless keels and cemented tibial trays: 10-year results. Int Orthop 2009;33(1):117-21.

[41] Sehat KR, Evans RL, Newman JH. Hidden blood loss following hip and knee arthroplasty. Correct management of blood loss should take hidden loss into account. J Bone Joint Surg Br 2004;86(4):561-5.

[42] Ares O, Seijas R, Sallent A, Popescu D, Lozano L. Bleeding in Knee Arthroplasty: Age, Drained Volume, and Ischemia Time. J Knee Surg 2015;28(4):329-34.

[43] Gao FQ, Li ZJ, Zhang K, Huang D, Liu ZJ. Risk factors for lower limb swelling after primary total knee arthroplasty. Chin Med J (Engl) 2011;124(23):3896-9.

[44] Committee WotAAoHaKSEB. Obesity and total joint arthroplasty: a literature based review. J Arthroplasty 2013;28(5):714-21.

[45] Jørgensen CC, Kehlet H, Group LFCfF-tHaKRC. Fall-related admissions after fast-track total hip and knee arthroplasty - cause of concern or consequence of success? Clin Interv Aging 2013;8:1569-77.

[46] Giusti A, Barone A, Razzano M, Pizzonia M, Pioli G. Optimal setting and care organization in the management of older adults with hip fracture. Eur J Phys Rehabil Med 2011;47(2):281-96.

[47] Rubin LE, Blood TD, Defillo-Draiby JC. Total Hip and Knee Arthroplasty in Patients Older Than Age 80 Years. J Am Acad Orthop Surg 2016;24(10):683-90.

[48] Walke LM, Rosenthal RA, Trentalange M, Perkal MF, Maiaroto M, Jeffery SM, et al. Restructuring care for older adults undergoing surgery: preliminary data from the Co-Management of 
Older Operative Patients En Route Across Treatment Environments (CO-OPERATE) model of care. J Am Geriatr Soc 2014;62(11):2185-90.

[49] Biboulet P, Bringuier S, Smilevitch P, Loupec T, Thuile C, Pencole M, et al. Preoperative Epoetin- $\alpha$ with Intravenous or Oral Iron for Major Orthopedic Surgery: A Randomized Controlled Trial. Anesthesiology 2018;129(4):710-20.

[50] Mat Eil Ismail MS, Sharifudin MA, Shokri AA, Ab Rahman S. Preoperative physiotherapy and short-term functional outcomes of primary total knee arthroplasty. Singapore Med J 2016;57(3):138-43.

[51] Calatayud J, Casaña J, Ezzatvar Y, Jakobsen MD, Sundstrup E, Andersen LL. High-intensity preoperative training improves physical and functional recovery in the early post-operative periods after total knee arthroplasty: a randomized controlled trial. Knee Surg Sports Traumatol Arthrosc 2017;25(9):2864-72.

[52] Wang L, Lee M, Zhang Z, Moodie J, Cheng D, Martin J. Does preoperative rehabilitation for patients planning to undergo joint replacement surgery improve outcomes? A systematic review and meta-analysis of randomised controlled trials. BMJ Open 2016;6(2):e009857.

[53] Masaracchio M, Hanney WJ, Liu X, Kolber M, Kirker K. Timing of rehabilitation on length of stay and cost in patients with hip or knee joint arthroplasty: A systematic review with meta-analysis. PLoS One 2017;12(6):e0178295.

[54] Malviya A, Martin K, Harper I, Muller SD, Emmerson KP, Partington PF, et al. Enhanced recovery program for hip and knee replacement reduces death rate. Acta Orthop 2011;82(5):577-81. [55] Mahomed NN, Davis AM, Hawker G, Badley E, Davey JR, Syed KA, et al. Inpatient compared with home-based rehabilitation following primary unilateral total hip or knee replacement: a randomized controlled trial. J Bone Joint Surg Am 2008;90(8):1673-80.

[56] Smith TO, Dainty JR, MacGregor AJ. Changes in social isolation and loneliness following total hip and knee arthroplasty: longitudinal analysis of the English Longitudinal Study of Ageing (ELSA) cohort. Osteoarthritis Cartilage 2017;25(9):1414-9.

[57] Lampley A, Huang RC, Arnold WV, Parvizi J. Total joint arthroplasty: should patients have preoperative dental clearance? J Arthroplasty 2014;29(6):1087-90.

[58] Andersson C, Wissenberg M, Jørgensen ME, Hlatky MA, Mérie C, Jensen PF, et al. Agespecific performance of the revised cardiac risk index for predicting cardiovascular risk in elective noncardiac surgery. Circ Cardiovasc Qual Outcomes 2015;8(1):103-8. 


\section{Tableaux}

Tableau 1. Bilan pré-anesthésique

\begin{tabular}{|c|c|}
\hline Bilans préopératoires & Objectifs \\
\hline \multicolumn{2}{|l|}{ systématiques } \\
\hline - hémogramme & Recherche d'une anémie préopératoire \\
\hline - groupage sanguin avec recherche d'agglutinines irrégulières & Bilan prétransfusionnel \\
\hline - électrocardiogramme 12 dérivations ( $\mathrm{si}>65$ ans) & Bilan cardiaque minimal \\
\hline \multicolumn{2}{|l|}{ recommandés } \\
\hline - bilan d'hémostase & Chirurgie hémorragique \\
\hline - fonction rénale avec mesure de la clairance de la créatinine & Anticiper la prise d'AINS en postopératoire \\
\hline - consultation de cardiologie & Fortement recommandée avec calcul du score de Lee \\
\hline \multicolumn{2}{|l|}{ non systématiques (selon l'examen clinique) } \\
\hline Bilan dentaire & Recherche de foyers infectieux chroniques \\
\hline Examen cytobactériologique des urines & Recherche d'une infection urinaire \\
\hline - échographie cardiaque & $\begin{array}{l}\text { Si score de Lee }>1 \text { ou patients symptomatiques } \\
\text { (dyspnée, insuffisance cardiaque, souffle systolique } \\
\text { non connu ou suspicion d'HTAP) }\end{array}$ \\
\hline - échoDoppler des troncs supra-aortiques & si suspicion clinique ou antécédents \\
\hline - épreuve d'effort / scintigraphie myocardique & en fonction de la consultation cardiologique \\
\hline - radiographie de thorax & si pathologie pulmonaire évolutive connue \\
\hline \multicolumn{2}{|l|}{ Evaluation des traitements } \\
\hline - antihypertenseurs (IEC)* & Prévention de la dégradation de la fonction rénale \\
\hline
\end{tabular}




\begin{tabular}{l|l}
\hline - antiagrégants plaquettaires* (hors aspégic) & Prévention du risque hémorragique \\
- anticoagulants oraux (HBPM ou AVK)* & Prévention du risque hémorragique \\
- antidiabétiques oraux* & Prévention des troubles de la glycémie \\
\hline$*$ traitements devant être arrêtés ou modifiés en préopératoire.
\end{tabular}

* traitements devant être arrêtés ou modifiés en préopératoire.

IEC : inhibiteur de l'enzyme de conversion ; HBPM : héparines de bas poids moléculaire ; AVK : antivitamine $\mathrm{K}$ 


\section{Légendes}

Fig. 1. Prise en charge chirurgicale d'une coxarthrose droite (A) par arthroplastie totale de hanche utilisant un cotyle rétentif permettant de réduire le risque de luxation de prothèse.

Fig. 2. Radiographies postopératoires de face (A), de profil (D) et défilé fémoro-patellaire (C) d'une prothèse totale de genou droit. En cas d'ostéoporose, il est recommandé d'utiliser une quille d'extension tibiale pour améliorer l'ancrage de la pièce tibiale et limiter le risque d'enfoncement ou de descellement précoce de l'embase tibiale. 


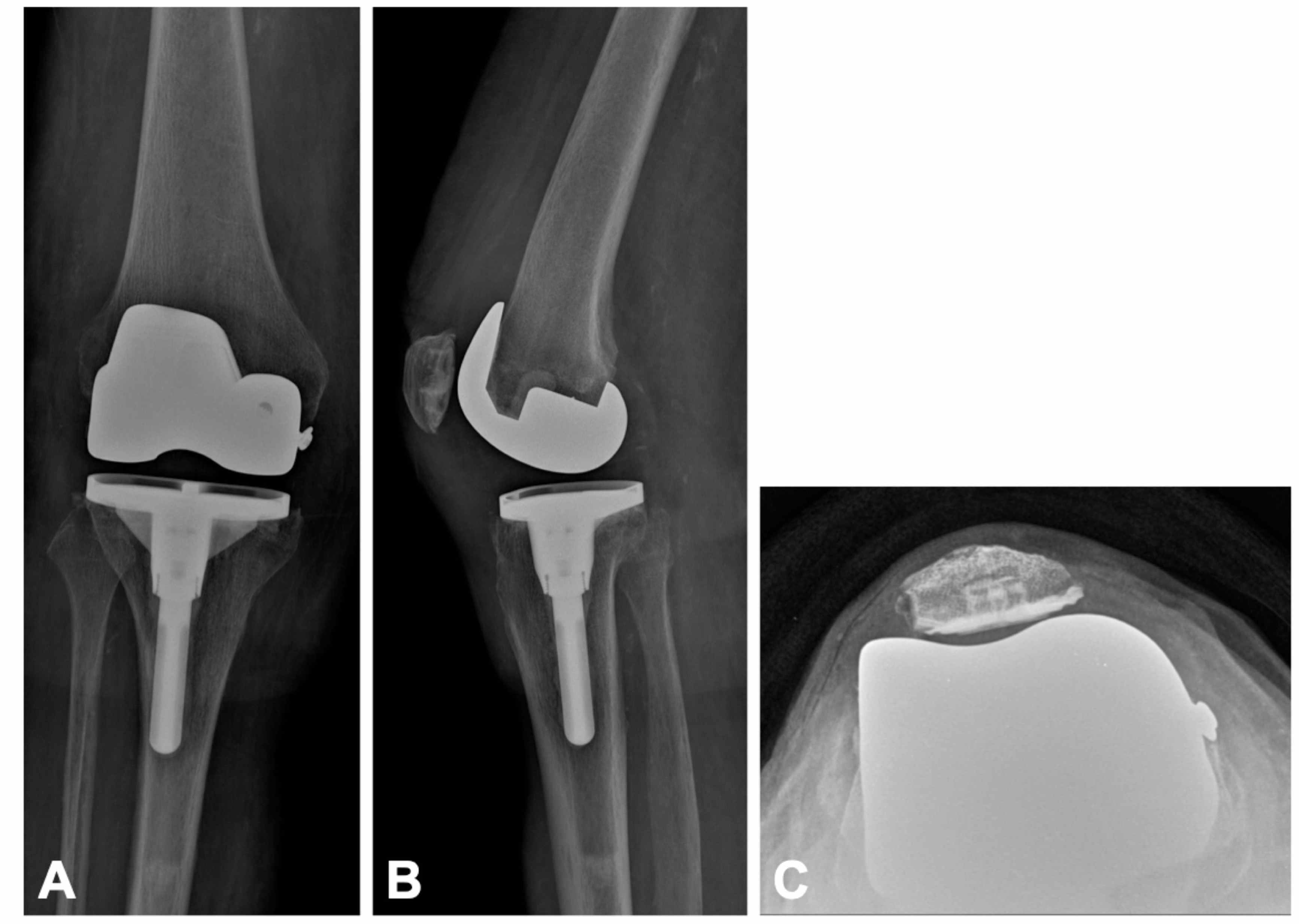

